\title{
Study on the Change of the Tone in the Flute Indoor Performance
}

\author{
Kuan Zhang \\ Xi’an Conservatory of Music, Xi’an, Shaanxi, 710061
}

Keywords: Tone Change, Flute, Control Charts, Indoor Performance

\begin{abstract}
Flute This unique musical instrument is not only played an important role in the orchestra, but also widely used in chamber music. In the chamber music, the flute because of its soft and soft tube of the sound, both with the wind instrument harmony, but also complement each other string instruments. In the form of rich performance, the player how to the flute's unique voice to take the initiative to control, in order to achieve with the common performance of the instrument sound more fusion purposes, is very worth exploring. Because of the complexity of multiple attributes, it has been more difficult to elaborate, so by layer analysis of the factors that determine the tone, and then explain the purpose and means of flute tone change is more academic significance. This article from the acoustical point of view and music psychology point of view to analyze why, and how to play in the chamber music will be changed to the flute sound.
\end{abstract}

\section{Introduction}

There are many forms of chamber music related to flute, and it is necessary to understand the particularity of the flute before dealing with the problem. Flute can be said to be one of the oldest known instruments, and its origin can be described in the Stone Age. The originator of the modern flute is the reed flute from Asia to the West. In 1100, the flute first appeared in the picture of Germany. Early flute with wood drilling production, shape and China's bamboo flute similar to the sound effect is similar to the Chinese Xiao sound. 1680 before and after, because of the requirements for the long range and pitch, the French for the first time for the flute fitted with a button, which was identified as the early flute to the beginning of the evolution of modern flute. Beginning in 1812, the German flute player Pomer (Theobald Boehm, 1794 1881) on the basis of predecessors on the flute for further transformation. Fifteen years later, in 1847, Pomer showed the first modern flute in front of the public. The flute's wall was made of metal, and the sound was much larger than the traditional flute. There is a key, and by mechanical linkage 3. This flute is named "Boehm flute" (Boehm flute), which is characterized: compared to the traditional flute, Pum flute's pitch has been greatly improved; thin wall, so that the flute vibration more Easy, so the limit volume is greater; sound more pure and bright; due to a variety of keys and add the key to add, the range is more extensive. At this point, the flute basically completed the transformation of the shape, in addition to the subsequent drop B control rod, today's flute is almost entirely used in 1847 Pomer's design.

\section{Flute of the Ensemble Form}

The accompaniment of the same kind of instrument has always been the most basic form of the ensemble, the flute's ensemble form has multiple flute, flute and Piccolo, medium and bass flute combination form, and even flute orchestra. Due to the relative unity of the sound, this type of music played by the ensemble will be very harmonious, as long as the play to pay attention to reduce the performance methods and habits of the differences can be caused by the representative works for Franz Doppler Flute Duo "Lineboard and Rondo" (Franz Doppler, Andante and Rondo).

Flute and other instruments of the combination of chamber music, the most important is the wooden quintet, the standard allocation is flute, oboe, clarinet, horn and large tube of a. Wood 
quartet and string quartet of the establishment of the period is similar to the formation of the classical music in the late 18th century and early 19th century, but because of the woods of the various musical instruments, the form of wooden quintet is not like a string quartet By some great classical musicians composer attention, therefore, this period did not leave a lot of excellent representative works. But with the subsequent completion of the restructuring of woodwind, woodwind instrument expression and playing skills to an unprecedented height, the composers began to increasingly like the use of wooden quintet in the form of some outstanding works gradually emerged. This combination of forms, because the instrument is playing with the wooden group of musical instruments, so the sound is more fusion, but the sound between the various instruments are different, so compared with the simple flute, need to tone in the unity of slightly Some of the works of the more representative works are Iber (Ibert) "for the wooden quintet made three pieces" and so on.

In addition to the combination with the wind instrument, the flute is often played with the stringed instrument, the more representative is Mozart for the flute chord instrument group written by the flute quartet (works K.285, K.285a, K.285b and K.298) The The configuration of the flute quartet is very similar to the string quartet, except that the position of the first violin is replaced by flute. In addition, there are many adapted from the string quartet of the flute quartet works, also are the flute instead of the first violin playing. This form of playing in the tone of the unity is more difficult, flute and stringed instruments because of the different structure, the pronunciation of different principles, playing different methods have resulted in a relatively large difference between the tone, more prone to tone separation of the situation, that listener Think flute and string is in self-care to play, there is no strong sense of integration. Therefore, when playing with the string, the flute player needs to focus more on the sound fusion.

In addition to the above forms, the flute will also play with a variety of musical instruments, such as flute and guitar (Carnon's "Nocturne" Op.109) or flute with the cello, piano combination (Weber's "Sonata") Wait. With the gradual enrichment of composing techniques, composers have become increasingly demanding for the fusion of many musical styles, and the seemingly unbelievable combination has begun to appear more and more, such as the combination of flute and electro-acoustic instruments, flute and traditional Chinese musical instruments Of the combination and so on. As a result, the flute in the chamber music playing in the tone change is particularly important.

\section{Music Interpretation Style and the Characteristics of the Times on the Flute Sound Requirements}

Here the classical music refers to the classical music, romantic music, etc., and modern music is different from the style characteristics. Classical chamber music requires flute playing to follow our own impression of the flute sound. That is, classical music to romantic early works, the sound of wood texture strong, soft and delicate voice; romantic after the music works are shining metallic luster, misty and Smart. Such as Juliani's "flute and guitar nocturne"

When the world of national music began to blend and learn from each other, many local characteristics of dance elements began to appear in the chamber music works, on behalf of the works of Piazzolla "tango history." This type of work is characterized by regional or ethnic characteristics highlighted, with a strong identification, so the requirements of the flute tone according to the different style of work and national characteristics to adjust.

With the progress of the times, more and more modern music, or popular music at that time, the flute playing the sound has a new request. At the end of the nineteenth century, in the Impressionist music that sprouted in Europe, the harmony was completely obeying the sound, and the flute also found a new sound that was different from the rounded bright colors of classical music. Impressionist music to create a mysterious and misty atmosphere, so when playing, the flute need to play more divergent, unreal sound effects. In the 1930s, the rise of jazz in the use of flute music, and as a special effect of music and through the always saxophone, bass, percussion, piano and other interactive and exchange. In the jazz music flute is different from the classical chamber music 
flute sound soft and delicate, but in the mutual music level to reflect a slightly less than the main instrument (saxophone, guitar, etc.) and have the personality of the second subsidiary melody. In the sound, showing a slightly or insolent or loose or free emotional catharsis, requiring the player to change the traditional way of classical play, exaggerate the characteristics of some special effects and skills decorative, rich connotation for jazz music Unique music service. And later the rise and widespread popular rock and roll, also began to use the flute characteristics of the sound, as a fusion of public aesthetic and try, collide and burst into a new spark. Especially in the heavy metal and death rock style, boldly flute traditional character changes, a noisy, brutal, hysterical tone try, the public aesthetic habits to be reversed in order to achieve the rock music advertised mix and match, insolent, overturned Effect. In addition, in recent years, popular music is also a lot of use flute, but mainly as accompaniment instruments appear in the prelude or interlude, the sound according to the different styles to the diversity, but in the final analysis can not be strictly incorporated into the discussion of the chamber music Category, do not repeat them.

\section{Chamber Music Playing Flute Sound}

As we all know, the dominant voice is the frequency of sound waves, and the sound frequency is determined by the material and shape of the sound source, that is, the basic sound of a particular instrument is fixed. But why is the rapid development of technology in today's case, the synthesizer issued by the simulation of a variety of musical instruments, or so easy to be heard with the real instrument sound difference? About a century ago, C. Stryff 6 pointed out that if a sound "cut off" its head and tail, it would lose his character. And then some people have actually practiced C. Sen's this argument: the various instruments playing the loudness and volume of the same sound cut off the sound head and sound tail, play to the audience to listen to, so that they distinguish what kind of instrument playing, the results of the vast majority of the sound will be mistaken. In other words, although the synthesizer has completely replicated the spectrum of various musical instruments, it is easy to find that the sound emitted by the electronic musical instrument is not the sound of the real instrument because of the neglect of the characteristics of the composer.

Through this experiment, we can get two conclusions: a. Identify the key to the instrument sound is the beginning and termination. b. If the sound head (sound) or the sound tail (radio) using a special way to play closer to each other sound, then the wind instrument and musical instruments of the harmonica is a great possibility. We can use the violin as a string of musical instruments on behalf of the trumpet as a representative of the brass group instrument, flute as a representative of the wooden group instrument to analyze the state of each group of musical instruments. Let's take a look at the spectrum of the sound conditions of the standard sound a1 $(440 \mathrm{~Hz})$ played in the usual way (with no special playing method).

Strength, and pitch, time value, speed, is the composer in the spectral surface marked the most clear one of the elements of music, musicians are also the most important elements of the spectrum. With the development of music style, to the modern, the intensity of modern music in the important position has been highlighted, the composers for the classic strength of the symbols or marks are no longer satisfied, more and more committed to creating more complex, a more detailed method of intensity labeling.

In addition to the above two decisive points, there is a playing method will have a significant impact on the sound, have to talk about, that is, vibrato (Vibrato). The tremolo referred to here refers to a vocal sound effect that is caused by a slight change in the regular period of pitch and volume. The vibrations in the instrumental play are mainly the natural fluctuations in the sound of the vocal singing, the high frequency and the obvious change in the volume of the sound will make the music more sound, the sound is more bright and full, and the frequency is low and the volume change is small. So that listening to feel soft and warm sound, so the rational use of vibrato can make musical instruments played more singing and appealing. The tremolo played in the wind is called the vibrant trembling, which, like the vibrato in the vocal music, depends on the change of the atmosphere, and the corresponding playing method in the string is rubbing the string. Flute playing in the main use of trembling trembling, in the case of need will also be combined with chest 
tremor and throat. The specific method of playing the breech is: after preparing the breath, the diaphragm and the abdominal muscles for the rhythm of the squeeze, so that the flow of gas into a state of law fluctuations, resulting in the sound volume and pitch on the regular fluctuations. The use of lip tongs in brass instruments is to avoid the use of flute playing, because the lips of the chatter will lead to airflow direction disorder, so that the flute sound changes.

\section{Conclusion}

Chamber music is the essence of the combination of confrontation, with the tone is not the same or even very different instruments to complete a musical instrument, but the whole instrument into a totally natural, which requires players not only have profound music appreciation, but also have the ability to control the musical instrument changes. Self-adjusting tone is a combination of understanding, understanding, control of the three types of ability to combine the comprehensive ability, which greatly reflects the player's playing level and musical accomplishments.

\section{References}

[1] Yan Qi. From the flute's sound source and resonant body on the principle of its pronunciation [J]. Yuefu new sound (Shenyang Conservatory of Music). 2009 (01)

[2] Wang Youhua. On the multiple dimensions of the composition of the sound [J]. Chinese music. $2009(01)$

[3] Wang Yiru. Play in the control of the tone of the control [J]. Musical instruments. 2008 (09)

[4] Chai Qingwei. From the physical properties of the sound tone on the internal characteristics of the characteristics of sound effects [J]. Education and text (in late) .2007 (01)

[5] Park Changtian. Berlin wood tube quintet to bring our chamber music teaching thinking [J]. Musical instruments. 2003 (01) 